\title{
Optical Detection of Dust and Scratches on Photographic Film
}

\author{
GIORGIO TRUMPY and RUDOLF GSCHWIND, Digital Humanities Lab - Universität Basel
}

\begin{abstract}
Today's information society needs efficient and economic solutions for the digital restoration of the photographic heritage. Different methods have been adopted up to now for the automatic detection of dust and scratches; each method has pros and cons, and a limited field of effectiveness. The use of infrared radiation and the spatiotemporal image analysis are among the most effective methods, although they have their limits. The infrared radiation only works for dye-based material, while the spatiotemporal image analysis is not applicable for still images and is limited due to motion in the scene. The present work defines in detail a set of methods for optical dust and scratches detection applicable on any type of transparent photographic material (silver-based as well as dye-based material, still images as well as moving images). The term "optical" refers to the fact that the considered methods seek physical evidence of the presence of foreign bodies or irregularities on the film; this allows avoiding the typical digital artifacts produced by "nonoptical" methods, for which certain elements of the scenes are erroneously obliterated because they resemble dust grains or scratches. "PDD" (Polarized Dark-field Detection) detects the flaws using an image acquired in a polarized dark-field setup; "DCD" (Dual Collimation Detection) takes advantage of the Callier effect to locate the flaws; " $n$-MDD" (Multiple Direction Detection) entails the acquisition of $n$ images in dark-field setups with different directions of illumination, and the extraction of the differences between the images through multivariate analysis. A numerical evaluation of the performances of the MDD method with an eightfold acquisition (8-MDD) is carried out by comparing its flaw detection with the flaw detection provided by commercial software based on spatiotemporal image analysis.
\end{abstract}

Categories and Subject Descriptors: Applied Research [Research paper]

General Terms: Design, Experimentation, Performance

Additional Key Words and Phrases: Computational photography, digital restoration, photographic film, scattering

ACM Reference Format:

Giorgio Trumpy and Rudolf Gschwind. 2015. Optical detection of dust and scratches on photographic film. ACM J. Comput. Cult. Herit. 8, 2, Article 7 (March 2015), 19 pages.

DOI: http://dx.doi.org/10.1145/2597894

\section{INTRODUCTION}

Two main courses of action can be taken by archivists and curators to preserve their photographic collections: object- and content-oriented interventions. In case the contextual features are essential, a photograph should not be considered as a mere image, but rather as a "document" [Swan 1981]; in this case, the preservation effort must consider the material, focusing on the physical integrity of the film (object-oriented interventions). However, the concept of "original" is for photography more flexible than for other works of fine art [Benjamin 1963; Sassoon 2007]; the intrinsic reproducibility of photography

This work was supported by the Swiss National Science Foundation \#200021-130261.

Authors' addresses: G. Trumpy and R. Gschwind, Digital Humanities Lab - Bernoullianum, Bernoullistrasse 32, CH-4056 Basel; emails: \{giorgio.trumpy, rudolf.gschwind\}@unibas.ch.

Permission to make digital or hard copies of part or all of this work for personal or classroom use is granted without fee provided that copies are not made or distributed for profit or commercial advantage and that copies show this notice on the first page or initial screen of a display along with the full citation. Copyrights for components of this work owned by others than ACM must be honored. Abstracting with credit is permitted. To copy otherwise, to republish, to post on servers, to redistribute to lists, or to use any component of this work in other works requires prior specific permission and/or a fee. Permissions may be requested from Publications Dept., ACM, Inc., 2 Penn Plaza, Suite 701, New York, NY 10121-0701 USA, fax +1 (212) 869-0481, or permissions@acm.org.

(c) 2015 ACM 1556-4673/2015/03-ART7 $\$ 15.00$

DOI: http://dx.doi.org/10.1145/2597894 
has limited the importance of the physical essence of a photograph, and has shifted the focus of the preservation effort to its visual content (content-oriented interventions).

As a matter of fact, since long before digital technologies, "preservation" of motion pictures was synonymous with "duplication": The film itself was considered just a "carrier," and the carried image could be copied onto a new support when the original was damaged.

Digital restoration, which is the epitome of a content-oriented intervention, has acquired a stronger sense for photography and, as a result, digital methods for restoration of motion picture films have become more and more widespread [Gschwind et al. 1998; Bergman et al. 2008].

Today's information society needs efficient and economic solutions for the digital restoration of the photographic heritage [Wright and Williams 2001]. As the operations become more and more automated, restoration is becoming more affordable; this is especially the case for motion picture films, as a frame-by-frame manual restoration is extremely burdensome.

\section{DUST AND SCRATCHES DETECTION}

Dust and scratches are two completely different phenomena; nevertheless, they are very often categorized together. "Dust" may refer to any small foreign body that is accidentally lying on the film surface; it can be opaque, thus obscuring the underlying emulsion image, or conversely, it can have a certain degree of transparency. On the other hand, in the case of a "scratch," the film material is missing; scratches can be superficial or they may penetrate the emulsion layer, completely obliterating parts of the image impressed in it. A deep scratch on the side of the emulsion (whose thickness is generally around $10 \mu \mathrm{m}$ ) is likely to destroy the corresponding part of the photographic image.

The cause of both dust and scratches is mechanical; both dust and scratches represent irregularities of the film surface; both dust and scratches locally alter the image brightness. The term "flaw" is used in the present work referring indistinctly to the two phenomena.

The advanced solutions for the digital restoration of this type of flaw are carried out in two stages: detection and reconstruction. A well-done detection prevents unpleasing digital artifacts.

The task of the detection stage is to classify all the pixels of the digital reproduction in categories that assess the level of detriment or express the probability that the specific pixel is part of a flaw. When a binary classification is performed, the pixels are assigned to only two categories, "normal" or "flawed," and the result of the detection is a binary image: the detection mask. However, flaws often do not completely obliterate the underlying area; in light of this, the latest research in motion picture restoration often uses nonbinary opacity values [Elgharib et al. 2013].

The following inpainting operation is assisted by the detection mask, which indicates where the restoration is necessary and where the image has to be left untouched; the corrupted data of the digital reproduction are corrected so that the final image appears more pleasing and plausible to an average observer. Inpainting uses the information located near the boundaries of the degraded regions to restore continuous edges by connecting the truncated fragments together [Bertalmì 2006] and filling the lacunae by extending surrounding patterns [Kwatra et al. 2005]. When nonbinary opacity values are adopted, flaws are reconstructed using also their original data [Crawford et al. 2007].

Even though the final purpose of digital restoration is to eliminate the visual consequences of flaws, the initial stage of optical detection strives for the opposite task of emphasizing the flaws; this way, it is possible to acquire an image that is easily transformed into the binary detection mask through simple thresholding. This initial flaw emphasis is the core topic of the present work.

\section{NONOPTICAL AND OPTICAL DETECTION}

Numerous methods have been so far adopted for dust and scratches detection; the main distinction is between two classes: "image processing" and "optical" methods. 
The methods based on image processing operate on the acquired digital data, relying on the appearance of flaws. With this approach, the flaws are detected relying on their morphological characteristics (e.g., scratches being often vertical, long, and narrow), or taking advantage of the high correlation between adjacent frames of motion pictures [Storey 1985; Kokaram 1998; Buisson et al. 2003; Kokaram 2004; Bertalmì 2006]. As a matter of fact, thanks to the little difference between the contiguous frames (which is what produces the illusion of movement), a spatiotemporal image analysis can detect and remove dust grains, dirt spots, and similar flaws that appear on a single frame [Schallauer et al. 1999]. A wide selection of applications (e.g., Diamant, Furnace, DaVinci Revival) are available for the spatiotemporal image analysis of movie sequences in digital form.

The image processing methods do not require a special scanning hardware, thus reducing the cost of restoration; moreover, they are applicable on previously digitized motion pictures, without the need to rescan the film.

The principal limit of the spatiotemporal image analysis is its inapplicability for still images. In addition, failures occur, on the one hand, due to the motion of the scene, which causes false detections; on the other hand, the persistency of certain types of flaws may entail missed detections. Actually, the detection of vertical scratches, which are due to the transportation of the film and persist in the same position over several frames, is the topic of abundant scientific research [Joyeux et al. 1999; Bruni and Vitulano 2004; Güllü et al. 2006; Newson et al. 2012, 2013].

The optical methods for flaw detection adopt a completely different approach, seeking physical evidence of the presence of foreign bodies or irregularities on the film surface, thus preventing false detections; these methods [Edgar 1993; Battles et al. 1999; Mead 2007; Landon et al. 2009; Pintus et al. 2010] require a special machinery/setup ${ }^{1}$ that highlights the particular physical evidence investigated. The principal drawback of the optical methods arises in the case of flaws that were impressed in the destination film during previous processes of copying or printing; their corresponding blotches are just traces of flaws and they may be detectable by relying on morphological characteristics of their appearance, but they are not in any case detectable using an optical method.

The most effective optical method for dust and scratches detection uses the infrared radiation [Edgar 1993]. The scanners equipped with the Digital ICE technology take advantage of the transparency of dye-based photographic film in the infrared range above $850 \mathrm{~nm}$; dust and scratches often entail an attenuance of infrared radiation, therefore they appear in the infrared image as definite dark marks on a homogeneous background. Unfortunately, this method is inapplicable on silver-based film, as the image-forming silver particles are opaque to infrared radiation.

Another optical method for the removal of dust and scratches on silver-based material has been developed by the British company Cintel [Mead 2007]. Their special scanner is equipped with an integrating sphere, whose interior surface has a portion with variable reflectivity; this characteristic allows processing two different signals corresponding to the scattered and the unscattered fractions of light, and this can render the flaws less visible, while simultaneously preserving sharpness and contrast.

\section{FILM-LIGHT INTERACTION}

The emulsion of a processed film consists of a dispersion of image particles in gelatin; the image particles allow for the attenuance of visible light, which is the fundamental phenomenon of photography.

Let $J_{1}$ be the luminous flux measured by a light sensor in the presence of a film between light source and sensor, and $J_{o}$ be the luminous flux measured in the absence of the film. The attenuance $D$ is

\footnotetext{
${ }^{1}$ For this reason, they are also called "hardware-assisted."
} 


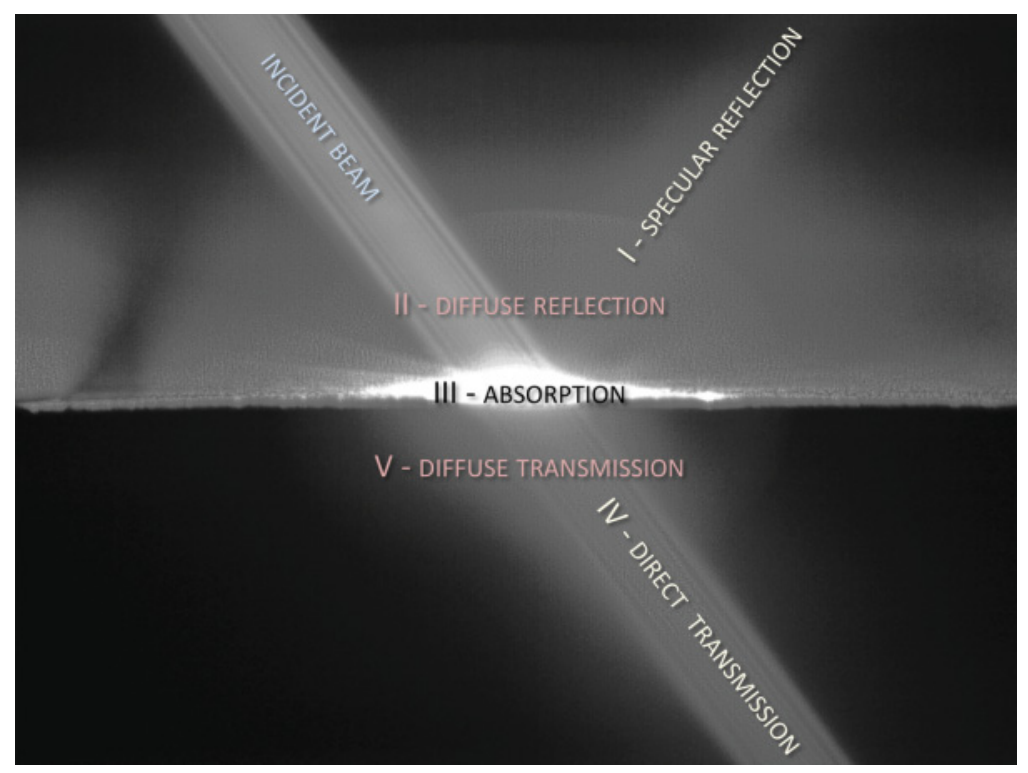

Fig. 1. Interaction between a thin pencil of light (the incident beam) and a silver-based film (the central horizon of the image).

defined as

$$
D=\log \frac{J_{o}}{J_{1}}
$$

The measured light is attenuated due to the depletion of the rays into the film (absorption) and due to their deviation in directions uncollectible by the light sensor (scattering) [Altman 1977].

Generally speaking, the image particles are metallic silver in black -and-white films and dye clouds in color films. A silver-based emulsion is a very heterogeneous system, in which silver particles typically span from $0.2 \mu \mathrm{m}$ to $2 \mu \mathrm{m}$ [Vitale 2009]; the optical characteristics of these particles are very different from those of the gelatin in which they are immersed, and therefore, they act as scattering centers [Bohren and Huffman 1983]. The illuminating rays are absorbed and scattered. On the other hand, the refractive indices of the dye clouds and of the gelatin are similar (at least the real part); hence, a wavelength-selective absorption is essentially the only phenomenon occurring in dye-based emulsions.

The overall phenomena occurring during the interaction between light and a photographic film can be classified as reported in Figure 1: The rays of the incident beam can be bounced backward, specularly (I) or diffusely (II), or they can be absorbed (III), or they can emerge from the other side, directly (IV) or diffusely (V).

In the case of a silver-based emulsion the diffused components (II and V) are remarkable, while in the case of color films the diffused components are smaller.

When imaging a photographic film, the directional properties of the illumination define the visual importance of the reflection, the transmission, and the scattering components of the film and its flaws [Hornberg 2006]; different features of the film will be emphasized in the image according to the illumination technique adopted, thus yielding varying appearances. The following sections report a set of illumination techniques that can be used in order to distinctly emphasize dust and scratches, thus being able to produce an exact detection mask. 

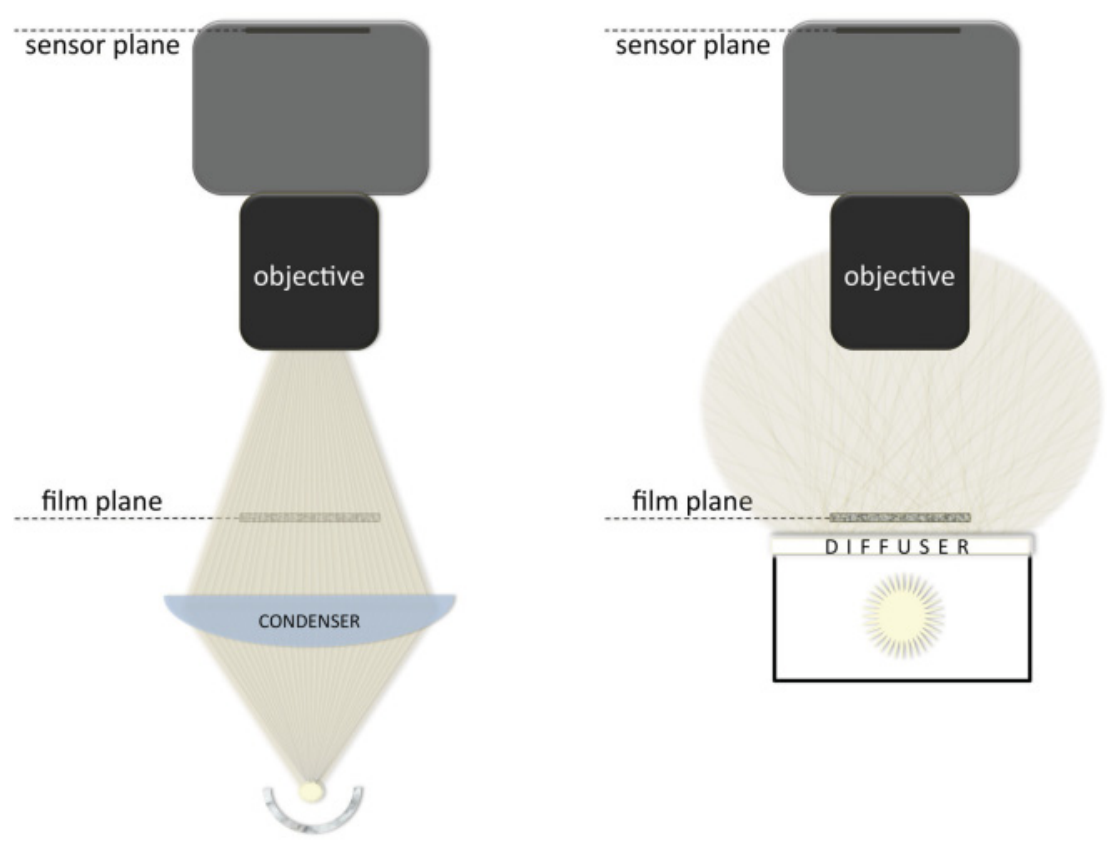

Fig. 2. Image acquisition with digital camera: Directed bright-field setup (left) and diffused bright-field setup (right).

\subsection{Bright-Field Setups-Callier Effect}

The term "bright field" refers to the most typical transmission setup and represents the operational setup for photographic transparencies, in which the illumination is paraxial (i.e., a substantial part of the rays that illuminate the sample do not diverge much from the optical axis of the imaging device). By varying the degree of collimation of the rays, it is possible to arrange different bright-field setups [Keeling 1972]: Two opposite manners are the directed (extremely collimated) and the diffused (extremely uncollimated) bright-fields (Figure 2).

The directed bright field has extremely strong directional characteristics by means of a point source and an optical system (condenser), making it the most energy-efficient illumination setup; ${ }^{2}$ in this case, each point of the photographic film receives light from only one direction. On the other hand, in a diffused bright-field setup, the light is scattered in an integrating chamber and the illumination is provided through a translucent slab (diffuser); in this setup, each point of the film receives light from all the directions.

The collimation of the illumination plays a fundamental role in the sharpness and contrast of the image impressed on a film [Tuttle 1926], and this is much more evident in the case of a silver-based material than for a dye-based material, as the latter has a far lower scattering fraction.

In case of relevant scattering fraction, the attenuance provided by the image particles strongly depends on the degree of collimation of the illumination. In Figure 3, the same silver-based film is reproduced in directed and diffused bright-field setups. The image on the left is much "crisper" and the film grain is emphasized together with dust and scratches; the image on the right appears "softer" and the details are smoothed. Moreover, the contrast on the left is much stronger than that on the right.

\footnotetext{
${ }^{2}$ In fact, it is adopted for projection in cinema theaters, where a high illuminance over a large surface is required.
} 

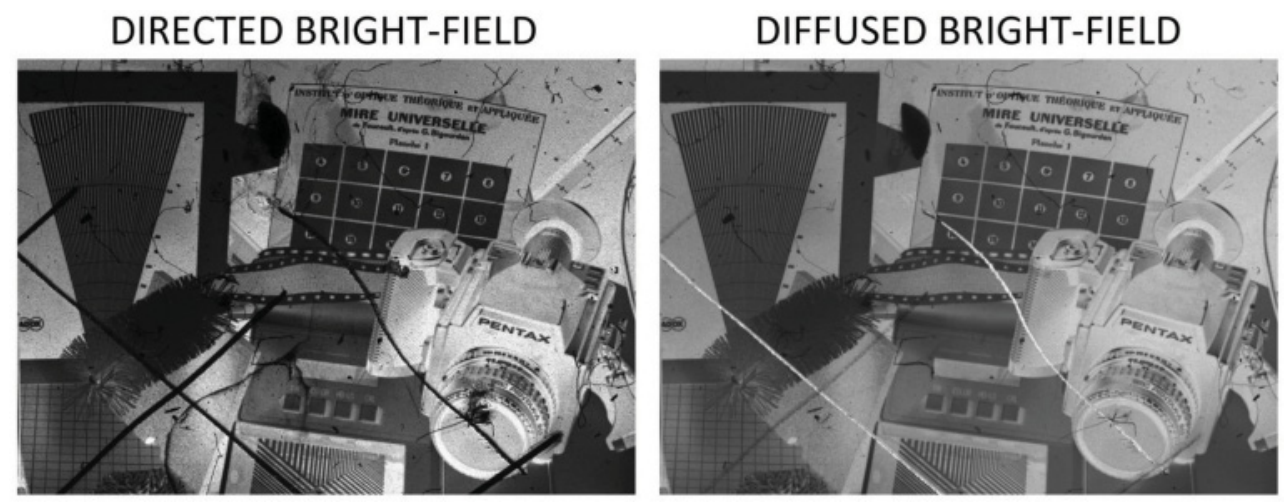

Fig. 3. Images of the same silver-based film acquired in directed and diffused bright-field setups.
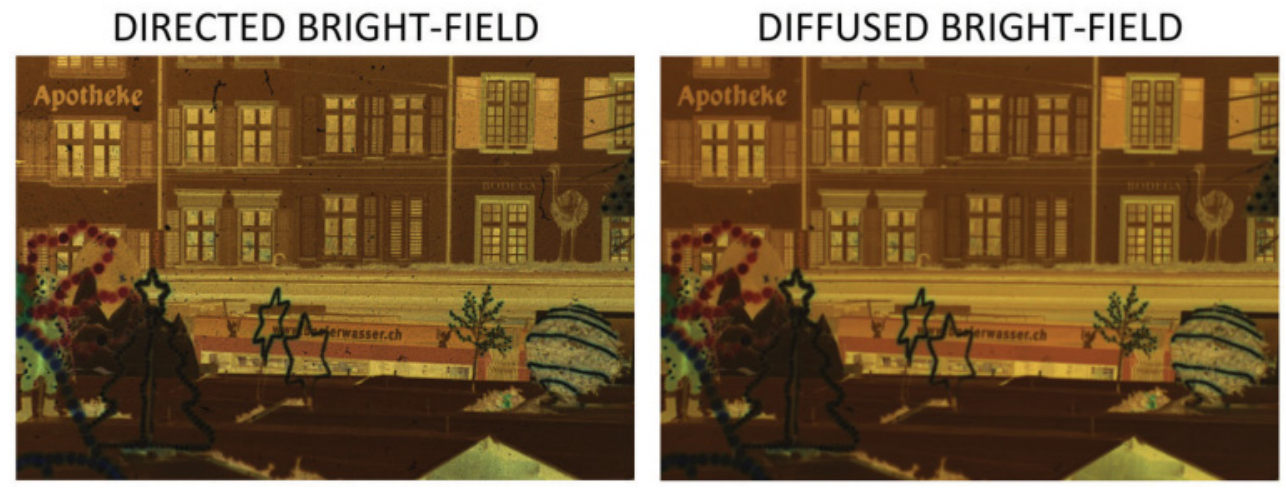

Fig. 4. Images of the same dye-based film acquired in directed and diffused bright-field setups.

In the case of dye clouds, the scattering fraction is small; the attenuance provided by the image particles is independent of the directional characteristics of the incident light. Figure 4 shows the images of a dye-based film acquired in directed and diffused bright-field setups; apart for dust and other imperfections that entail high scattering, the sharpness and the contrast is about the same in the two images.

The ratio between the attenuances provided by a specific point of a photographic film (refer to Equation (1)), measured in directed, $D_{d i r}$, and diffused, $D_{d i f}$, bright-fields, is termed the Callier $Q$ factor:

$$
Q=\frac{D_{d i r}}{D_{d i f}} .
$$

The Callier $\mathrm{Q}$ factor is always equal to or greater than unity; its trend versus the diffusely measured density $D_{d i f}$ is reported in Figure 5 for a typical silver-based film [Streiffert 1947]. On the small scale of the image structure, the direct effect of the $\mathrm{Q}$ factor is the variation in sharpness of the images produced illuminating with different light collimation; on the large scale of the entire image, the equivalent effect is the variation in contrast. These variations (for example, between the images of a condenser and a diffuser enlarger) have been observed over a long period of time [Callier 1909], and they became known as the "Callier effect." 


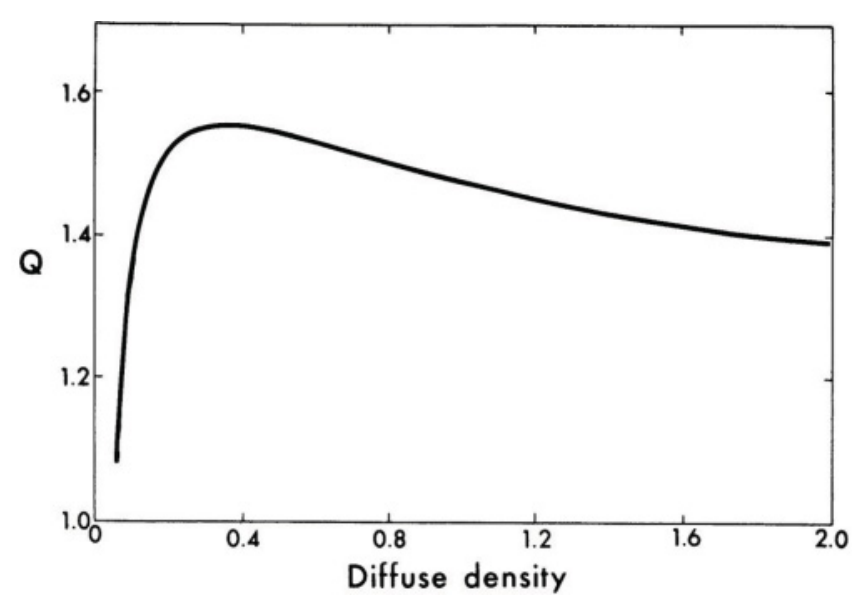

Fig. 5. Callier $\mathrm{Q}$ factor versus diffuse density for a silver-based film.

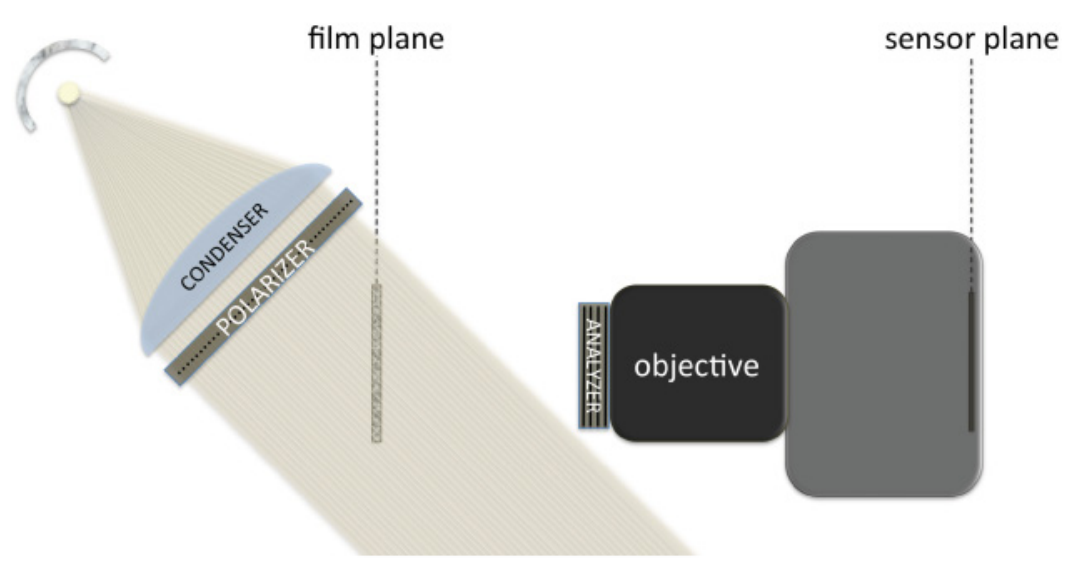

Fig. 6. Polarized dark-field setup for film reproduction.

\subsection{Polarized Dark-Field Setup}

The term "dark field" indicates a special illumination geometry in which the sample is illuminated obliquely, so that no directly transmitted light is collected by the optical system: only scattered light can be collected. Such an arrangement is useful to emphasize scattering elements [Battles et al. 1999].

Scattering can produce light with polarization characteristics different from those of the incident beam [Mishchenko and Travis 2000] and therefore, when illumination is linearly polarized in full, the scattered light may become partially or totally unpolarized; the phenomenon is called depolarization and it can occur even if the scattering particles are not optically active [Ghosh et al. 2004].

This phenomenon suggests combining a dark-field setup with polarizing filters, as depicted in Figure 6 [Gschwind 2010]; the use of a parallel light beam allows a uniform illuminance on the film. The dark-field setup is useful to emphasize structures, shape irregularities, and impurities in the film, such as dust and scratches; these elements generally depolarize light more than the photographic emulsion, and therefore, the utilization of linearly polarized light in a dark-field setup further emphasizes them [Trumpy et al. 2011], as shown in Figure 7. 

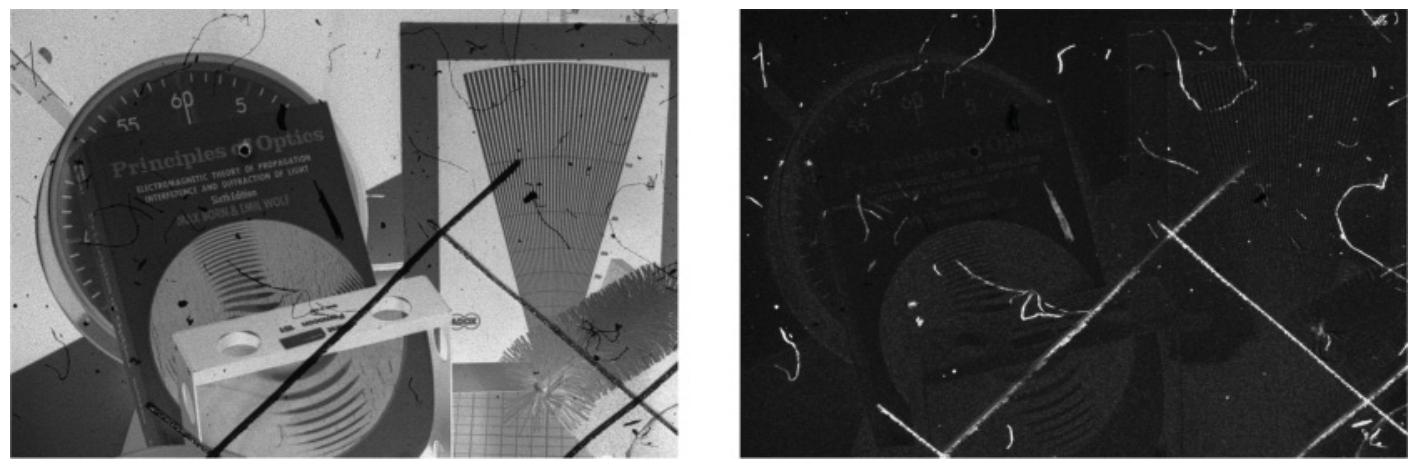

Fig. 7. A sample with dust and scratches illuminated in bright field (left) and in polarized dark field (right).

\section{CREATION OF DETECTION MASK}

Based on the illumination techniques described in Section 4, the present section defines in detail three innovative methods for optical dust and scratch detection, which are effective on any type of photographic film: both silver-based and dye-based material, as well as still images and moving images.

A simple binary detection is considered in the present work; regardless of flaw transparency, the pixels are assigned to "normal" or "flawed" categories. In prospect, the detection data provided with the presented optical systems could be also used to provide nonbinary opacity values.

\subsection{Polarized Dark-Field Detection}

The main parameters involved in the arrangement of a polarized dark-field setup (Figure 6) are the spectral emission of the sources, the orientation of polarization ( $S$-polarized, $P$-polarized), and the angle between the light and the optical axis.

A specific analysis was carried out to study the influence of these parameters on the efficiency of the dust and scratches detection [Trumpy et al. 2011]. In light of this "parameter analysis," the best arrangement was identified; it was demonstrated that the use of a broadband white light is the most convenient choice, and that the polarization orientation with respect to the film plane does not have any significant influence: that is, there is no favorite orientation between P-polarization and S-polarization. As for the angle between the beam of light and the optical axis, it has been found that the higher the angle, the stronger the emulsion/dust and scratches differentiation will be; so from this perspective, a higher angle should be preferred. However, it has to be taken into consideration that, as the angle gets larger, the light intensity collected by the imaging device gets lower and, hence, the exposure time gets longer; especially with a view to the application of the method to motion pictures, for which multitudinous frames have to be processed, the time necessary to accomplish the whole procedure may increase excessively. Moreover, longer exposures may entail a higher noise in the images. These contrasting considerations induce choosing a moderate angle around $45^{\circ}$.

In order to put the flaw detection into effect, a threshold-above operation is carried out on the image acquired in polarized dark field, thus creating the binary detection mask. The stronger the differentiation between the signal of the emulsion image and the signal of the flaws, the less critical the choice of the threshold value will be. ${ }^{3}$

We refer to the described method-acquisition of the polarized dark-field image and segmentation through a threshold—with the acronym PDD (Polarized Dark-field Detection).

\footnotetext{
${ }^{3}$ In many cases an adaptive thresholding may improve the flaw detection [Wallis 1976]. 


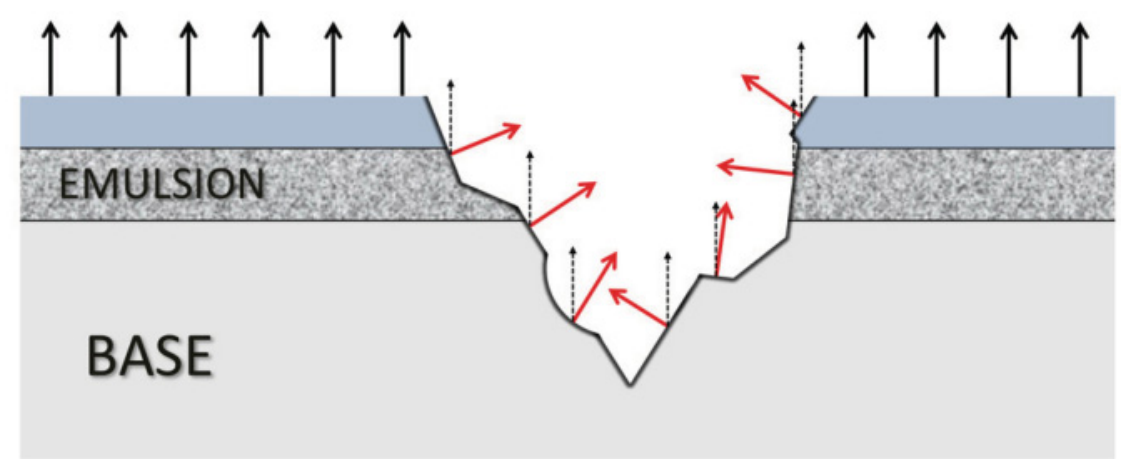

Fig. 8. The section of a film with a penetrating scratch. The red arrows indicate the local normal, which deviates from the intact film normal (dotted arrow).

\subsection{Dual Collimation Detection}

Due to the Callier effect (which was described in Section 4.1), in the image acquired in a directed bright field the film grain is emphasized together with dust and scratches, while, on the other hand, when a diffused bright field is adopted, the image has less sharpness and contrast, although the flaws are much less visible.

By acquiring the directed and the diffused bright-field images with a standard sampling density of $3000 \mathrm{ppi}$, the grain appearance in the image is barely resolvable, while dust and scratches are bigger and well visible. As a consequence, dust and scratches are the main differences between the two images, and therefore, the extraction of these differences is effective for flaw detection.

The image differences are positively extracted through a Principal Components Analysis (PCA). The flaw detection method that will be described in the next section (Section 5.3) also uses the difference extraction through PCA: The following Section 5.4 will explain this process, which is valid for both detection methods.

We refer to the just described method-acquisition of two images with different lighting collimations and computational detection through PCA-with the acronym DCD (Dual Collimation Detection).

\subsection{Multiple Direction Detection}

Dust and scratches on a film produce shadows and glows that change position according to the provenance of the light beam. When the order of magnitude of the flaw size is hundreds of microns or higher, by varying the direction of illumination in an image of 3000ppi, the position of glows and shadows of such flaws can shift by several pixels.

The differences between images acquired with different illuminating directions are related to the local deviation of the surface normal from the normal of the intact film surface (Figure 8). An irregularity in the surface, due to a scratch or a dust grain, can have any shape and, therefore, the deviation from the film normal for different points varies randomly. Every point of a flaw glows depending on the relation between the local surface normal and the direction of illumination.

This fact has already been used in agricultural engineering [Xiao 2010] for extracting superficial defects from sets of images of the same object acquired with different illumination. A similar approach has also been used for photographic prints [Pintus et al. 2010] and for films affected by Vinegar Syndrome [Landon 2013]. Image acquisition with multiple illumination directions is also the basis of Reflectance Transformation Imaging (RTI) techniques [Palma et al. 2010], which are of increasing interest in the cultural heritage field. 


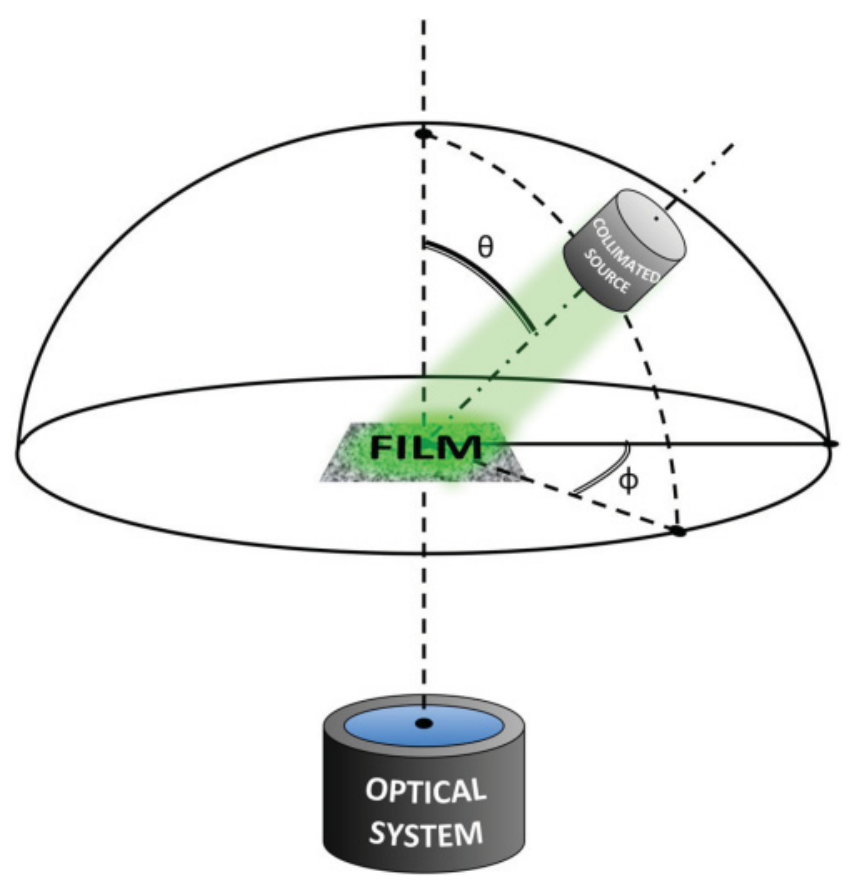

Fig. 9. The acquisition setup for the multiple direction detection.

A correct detection mask of a photographic film can be produced with multiple images acquired in a transmission setup with different directions of illumination (Figure 9), extracting the differences between the acquired images [Trumpy and Gschwind 2012; Rüfenacht et al. 2013]. We call this method Multiple Direction Detection, and we indicate it with the acronym $n$-MDD.

The main parameters involved in its arrangement are (I) the polarization of light and (II) the number $n$ of different directions included. A specific "parameter analysis" has been carried out to study the influence of these parameters on the efficiency of dust and scratches detection [Trumpy 2013]; in light of this parameter analysis, the optical setup presented in our previous works [Rüfenacht et al. 2013] has been optimized. In particular, the use of polarized light lengthens the acquisition times and does not provide a clearly better detection: ${ }^{4}$ since the runtime is a critical parameter, especially for motion pictures, we prefer to exclude the polarizers in this procedure. The "parameter analysis" has also shown that the greater the number $n$ of directions that are used, the more probable it will be that the flaws will be completely highlighted in all their parts; in fact, by using a larger number of illumination directions, it is likely that the suitable direction for each local deviation from the film normal (Figure 8) will be also included. However, it is necessary to bear in mind that the usage of a higher number of images implies a higher complexity in the acquisition stage and a more onerous processing.

In the present work, we consider eight directions adequate for the multiple direction detection (8MDD), in which the position of the light source is at polar angle $\theta=45^{\circ}$ and at azimuths $\phi=0^{\circ}, 45^{\circ}$, $90^{\circ}, 135^{\circ}, 180^{\circ}, 225^{\circ}, 270^{\circ}$, and $315^{\circ}$ (Figure 9 ).

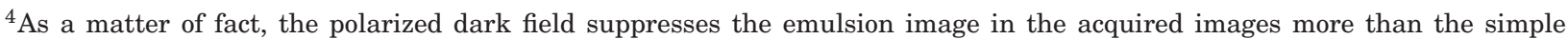
dark field; however, the subsequent computation provides a consistent suppression of the emulsion image in both polarized and unpolarized cases, and the performances of the flaw detection level off.
} 

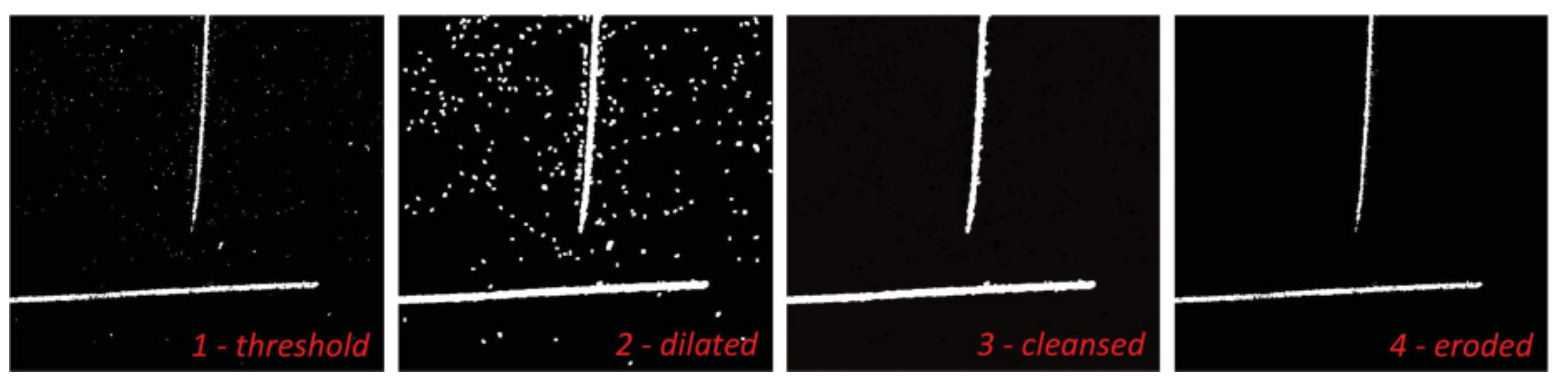

Fig. 10. Operations for the selection of the elements over a size threshold.

\subsection{Difference Extraction through PCA}

In many applications (e.g., image compression), PCA is used to reduce the dimensionality of a dataset, that is, selecting the eigenvectors with the highest eigenvalues [Gonzalez and Wintz 1987]. In our procedure, PCA is used to extract the differences between images acquired in different illumination conditions (i.e., different collimation in DCD (Section 5.2), and different directions in $n$-MDD (Section 5.3)) and thus, a different approach is adopted [Rüfenacht et al. 2013].

The output of PCA consists of $N$ images (eigenimages) that are the result of particular linear combinations of the $N$ input images. By applying the PCA on the set of eight images acquired for the accomplishment of the 8-MDD method (Figure 9), if we consider films in which dust and scratches affect a minor part of the frame (otherwise, any automatic unsupervised restoration would inevitably entail severe artifacts), the first eigenimage is found to have all positive components (as in the exemplifying matrix reported in Section 6.1). PCA's prerequisite of mutual orthogonality causes all the remaining eigenvectors to have a similar amount of positive and negative components; these eigenvectors actually take into account all of the differences between the original images, and these differences correspond to dust and scratches.

In view of the preceding dissertation, the first eigenimage is excluded (since the emulsion image remains visible) and the information from all of the remaining eigenimages (in the case of DCD, there is only one remaining eigenimage) is used for the differences extraction.

Contrary to the polarized dark-field images of PDD, the eigenimages require a different thresholding operation to create the binary detection mask. In this case, a threshold-outside operation (adaptive if needed) has to be carried out; the resulting binary images are added together in inclusive disjunction (logical OR) into a unique detection mask.

\subsection{Flaw-Size Threshold}

The binary images resulting from the thresholding operations so far described, in addition to the correctly detected flaws, may contain erroneous elements in the form of a spread of randomly distributed little points; these erroneous elements can be caused by the silver grain that was not completely suppressed, or by the electric noise in the acquired images. An effective solution to remove these randomly distributed points is to exclude the structures smaller than a certain area. The four-step procedure to accomplish this task is described with an example in Figure 10.

A Connected-Components Labeling (CCL) is performed on the rough binary detection mask (Figure 10-1 - threshold), and the elements are identified [Soille 2003]. A size threshold applied on the labeled elements would be sufficient to select the elements larger than a certain number of pixels and exclude the smaller ones ("cleansing"). However, it is often the case that not all the parts of a correctly detected flaw are connected together, and therefore, the CCL identifies the single flaw with 
multiple labels; in this case, the size threshold would also cleanse small unconnected parts of flaws that were actually correctly detected. To avoid this, a preliminary dilation prior to the size threshold is effective; the elements labeled in the first CCL are dilated to fill the fractures in the flaws (Figure 10-2 - dilated) and a second CCL is performed. Afterwards, the size threshold is applied to the elements (Figure 10-3 - cleansed). Eventually, it is necessary to perform an erosion to return to the undilated cleansed image (Figure 10-4 - eroded).

In addition to excluding the erroneous bright points, the cleansing operation can be considered a practical application of the principle of "minimal intervention." According to this principle of ethical restoration [FIAF 2013], the reconstruction should alter the original information only if absolutely necessary; as a matter of fact, when a flaw is very small, it does not truly compromise the proper use of an image and, therefore, it is better to leave the corresponding part untouched, rather than inpainting it with arbitrary information.

\section{DETECTION EVALUATION AND METHOD COMPARISON}

The numerical evaluation of a flaw detection is provided by the comparison between the binary "detection mask" and the binary image that indicates the "true" flaws of the sample: the "ground truth." In the present work, we consider it appropriate to compare the flaws detected by the automatic system under test with the flaws detected by an expert human observation; an operator creates the ground truth analyzing the bright-field image sequence and manually marking all the pixels that are judged flawed. Transparent and very small flaws have to be left out, as the proper fruition of the image is not seriously compromised [FIAF 2013].

From the pixel-to-pixel comparison between the detection mask and the ground truth, four cases are possible: (I) successful detection, (II) false detection, (III) missed detection, and (IV) void; the Matthews Correlation Coefficient (MCC) assesses the performance of the flaw detection with a unique number computed with the occurrences of these four cases [Baldi et al. 2000]. The MCC spans from -1 to 1 , and the higher it is, the better the detection.

Section 5 has defined in detail a group of methods for optical flaw detection applicable on any type of photographic film (silver-based as well as dye-based material, still images as well as moving images). The numerical evaluation of their flaw detection and the comparison of their performances have been carried out by the authors [Trumpy and Gschwind 2012], and the dust and scratches detection was found to be quite successful for all the methods. In light of the numerical results of this comparison, and in view of the state-of-the-art digital restoration, the present work considers the MDD a particularly innovative and promising technique.

\subsection{8-MDD vs. STIA}

A numerical comparison was carried out between the performances of the MDD with an eightfold acquisition (8-MDD-refer to Section 5.3) and the performance of the Spatiotemporal Image Analysis (STIA-refer to Section 3), which is the conventional approach for automatic dust and scratches detection on silver-based material.

The sample selected for the 8-MDD/STIA comparison is a $35 \mathrm{~mm}$ motion picture print film produced by the German company Lignose (mainly operative during the 1920s) [Kreimeier 1999]. Sixty-eight consecutive frames $(\simeq 2.8 \mathrm{~s}$ at $24 \mathrm{fps})$ of a static shot were considered in the experiment; the scene framed is an interior in which two characters perform a dance movement. The frames are severely affected by dust and scratches.

The digital images were acquired with a DSLR camera (Nikon D3) equipped with a 12Mpixel CMOS full-frame sensor. The reproduction ratio adopted was 1.2:1 (4-perf frame focused on a full frame sensor with margin) and the spatial sampling density was $3600 \mathrm{ppi}$; the image focusing was provided 
by a $105 \mathrm{~mm} \mathrm{f} / 4.5$ objective (UV-Nikkor) mounted on a bellow. White LUXEON K2 LEDs were used as light sources. The images were saved by the camera in the proprietary RAW format using 14bits; they were then externally converted in TIFF / 16bit format using the dcraw software [Coffin 2013], choosing the parameters to linearly translate the raw data.

For each of the 68 frames, nine $(8+1)$ perfectly registered images ${ }^{5}$ were acquired: eight dark-field images were acquired as depicted in Figure 9, using a light dome with a radius of $40 \mathrm{~cm}$; the other image is a reproduction acquired in diffused bright field (Figure 2, right).

Image stability is fundamental for an effective STIA [Schallauer et al. 1999]. In view of this, prior to detection and restoration, the motion created by the 68 frames was stabilized using the software NUKE (The Foundry Visionmongers Ltd); the static interior of the scene was considered as reference for the stabilization. The bright-field sequence was stabilized and the transformation found for each frame was applied equally to the eight dark-field images; finally, all nine images were registered and image sequence was stable.

Using the $F$ DirtRemoval plugin, the STIA was performed on the bright-field sequence, and a first set of detection masks was created; the "Very Aggressive" preset was selected, since "for archive footage you should typically choose Very Aggressive" [The Foundry 2011].

On the other hand, the detection masks of the 8-MDD method were created with an application (Scratch And Flaws detectoR and ANalyzer-SAFRAN) specifically developed for this project [Trumpy et al. 2011].

For example, the principal component analysis on the eight dark-field images of the 32nd frame produced the following orthogonal matrix:

$\left[\begin{array}{rrrrrrrr}0.1673 & 0.0052 & 0.1105 & 0.0146 & 0.3660 & 0.5309 & -0.1954 & -0.7111 \\ 0.1925 & 0.0088 & 0.1272 & 0.0616 & 0.7158 & -0.6287 & -0.1869 & 0.0168 \\ 0.1628 & 0.0033 & 0.1225 & 0.0172 & 0.3411 & 0.5484 & -0.2181 & 0.7026 \\ 0.1452 & 0.0132 & 0.0970 & 0.0141 & 0.2818 & 0.1085 & 0.9368 & 0.0183 \\ 0.5730 & -0.7287 & -0.3589 & -0.0048 & -0.1053 & -0.0256 & -0.0068 & 0.0023 \\ 0.3085 & 0.0489 & 0.4676 & 0.7774 & -0.2775 & -0.0445 & -0.0198 & -0.0090 \\ 0.3465 & -0.0082 & 0.6547 & -0.6216 & -0.2381 & -0.0846 & -0.0305 & -0.0069 \\ 0.5863 & 0.6828 & -0.4136 & -0.0696 & -0.1144 & -0.0258 & -0.0193 & 0.0044\end{array}\right]$.

The columns of the matrix express the eigenvectors. The data projected on the eigenvectors were rearranged in grayscale images, and these were used to produce the detection mask, following the procedure described in Section 5.4.

\subsection{Numerical Results of Comparison}

Each frame of the sequence was visually analyzed and its ground truth was created.

The SAFRAN software carried out the pixel-to-pixel comparison between the detection mask and the ground truth (Figure 11), and created the "detection-result" image (which is indexed to have four colors: successful detection—green, missed detection—red, false detection—blue, void—black); the MCC was then computed.

Figure 12 reports the MCC values for all the 68 fames, both for 8-MDD and NUKE. Apart for a couple of exceptions, the multiple direction detection outperformed the STIA for the entire analyzed sequence.

\footnotetext{
${ }^{5}$ All the images of a single frame were acquired without moving the camera or the film: only illumination manner changed.
} 

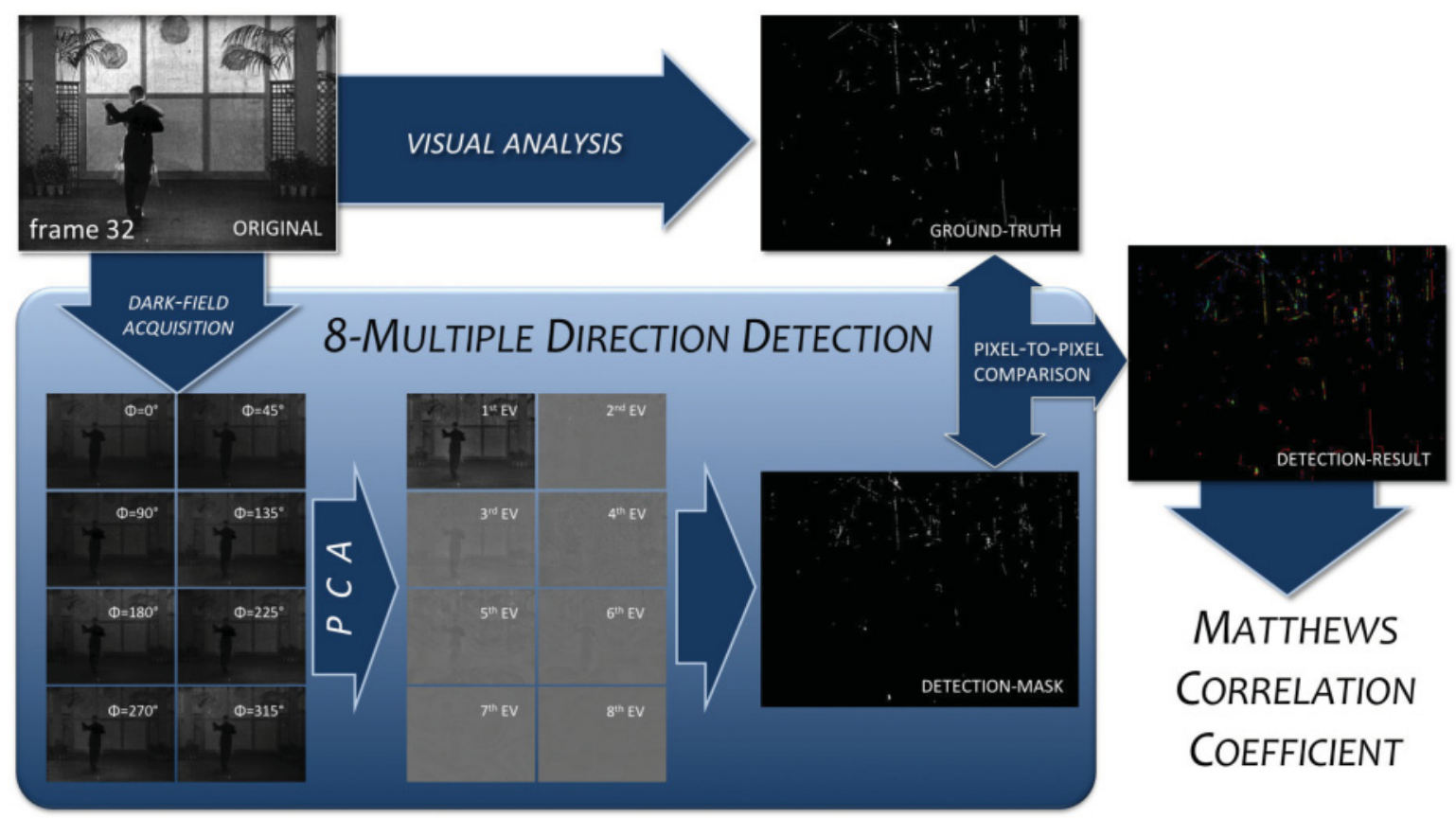

Fig. 11. The MDD on a single frame and evaluation of its result.

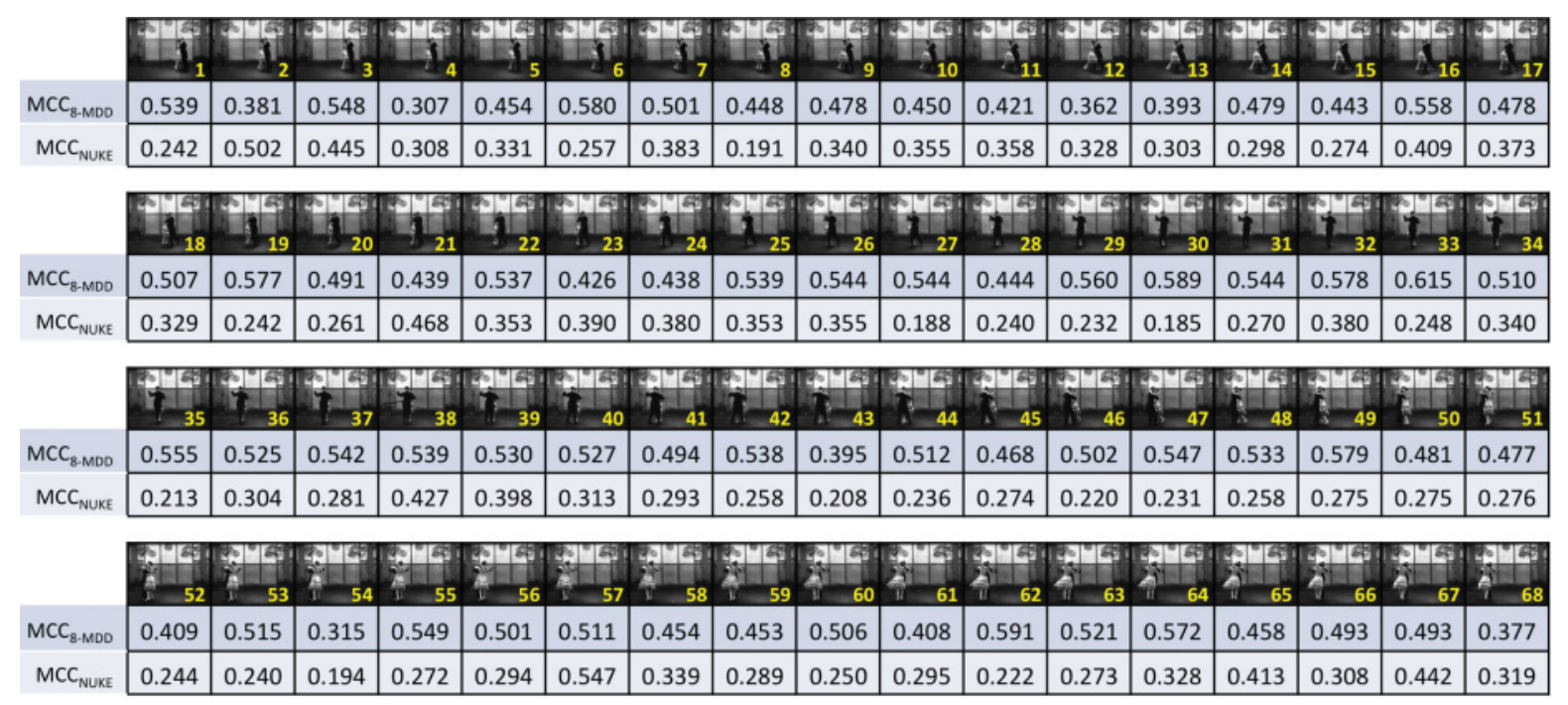

Fig. 12. MCC values of the STIA and of the multiple direction detection (8-MDD). 


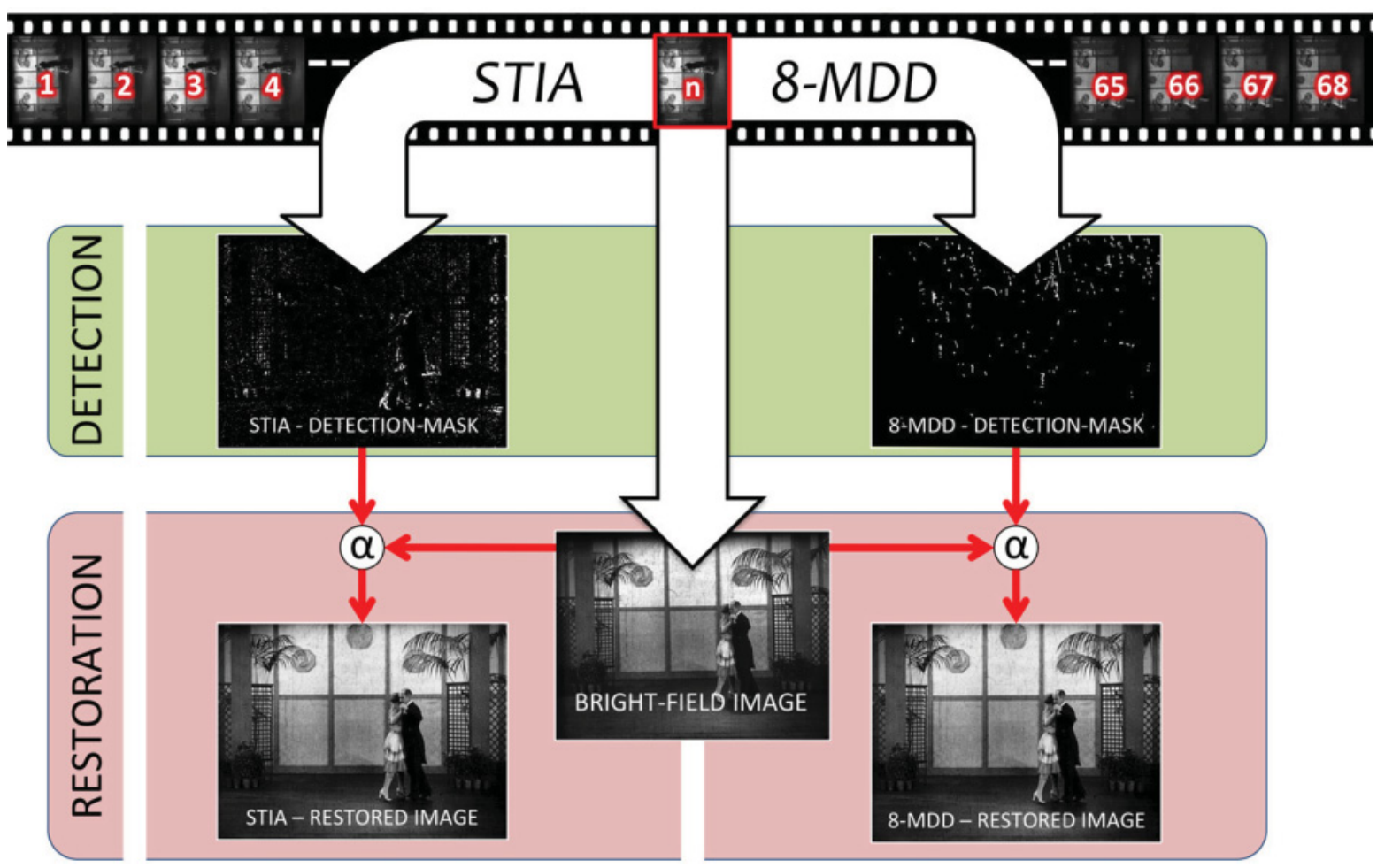

Fig. 13. Comparison between the restored sequences relying on the STIA and on the multiple direction detection (8-MDD).

\subsection{Qualitative Visual Comparison}

The two different detection masks were included in the alpha channel of each bright-field image of the sequence; this information provided "guidance" for the inpainting, which was performed for both detection masks by the NUKE's plugin F DirtRemoval (Figure 13).

The flaw detections and the two restored versions of the sequence were carefully examined; the following text reports the most relevant considerations that can be made analyzing the sequences.

The first rough impression is that the STIA version is cleaner than the 8-MDD version. As a matter of fact, STIA is a nonoptical method, and thus, it has been favored whenever the flaws were copied ones. Figure 14 reports the result of the flaw detection for the case of a copied flaw; the microscopic image clearly shows the silver structure of the blotch. In all these cases occurring in the sequence, the optical 8-MDD was deceived.

A more careful examination reveals the artifacts produced by STIA in correspondence to the moving elements of the scene. In the analyzed sequence, the position of the shoes of the dancers changes considerably between adjacent frames, and F DirtRemoval erroneously considered them as flaws.

Abundant scientific research has been carried out to develop algorithms that grasp the motion in image sequences and compensate it [Buisson et al. 2003; Corrigan et al. 2008]. F_DirtRemoval could accomplish the compensation only in the case of simple motion (e.g., uniform linear motion); in the case of the dance movement, several elements are moving in different directions and the compensation was impossible. Figure 15 reports the severe artifacts on one shoe of the dancers produced by the restoration based on the STIA. 

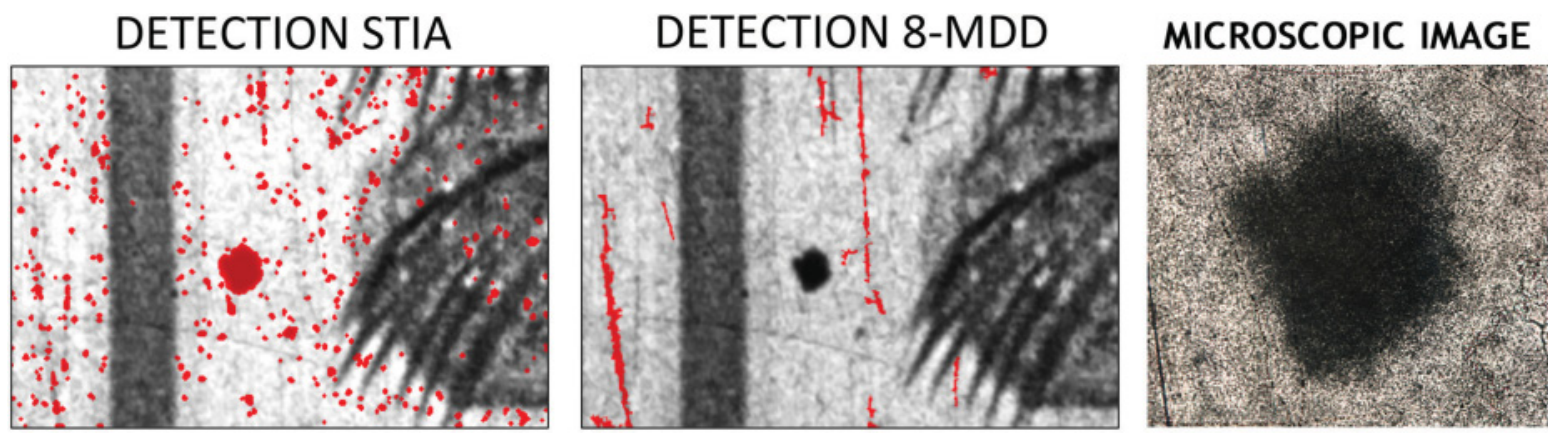

Fig. 14. Original image with detections highlighted in red: comparison between STIA (left) and 8-MDD (center). The dark blotch of the copied dust grain is enlarged through the use of a microscope (right).
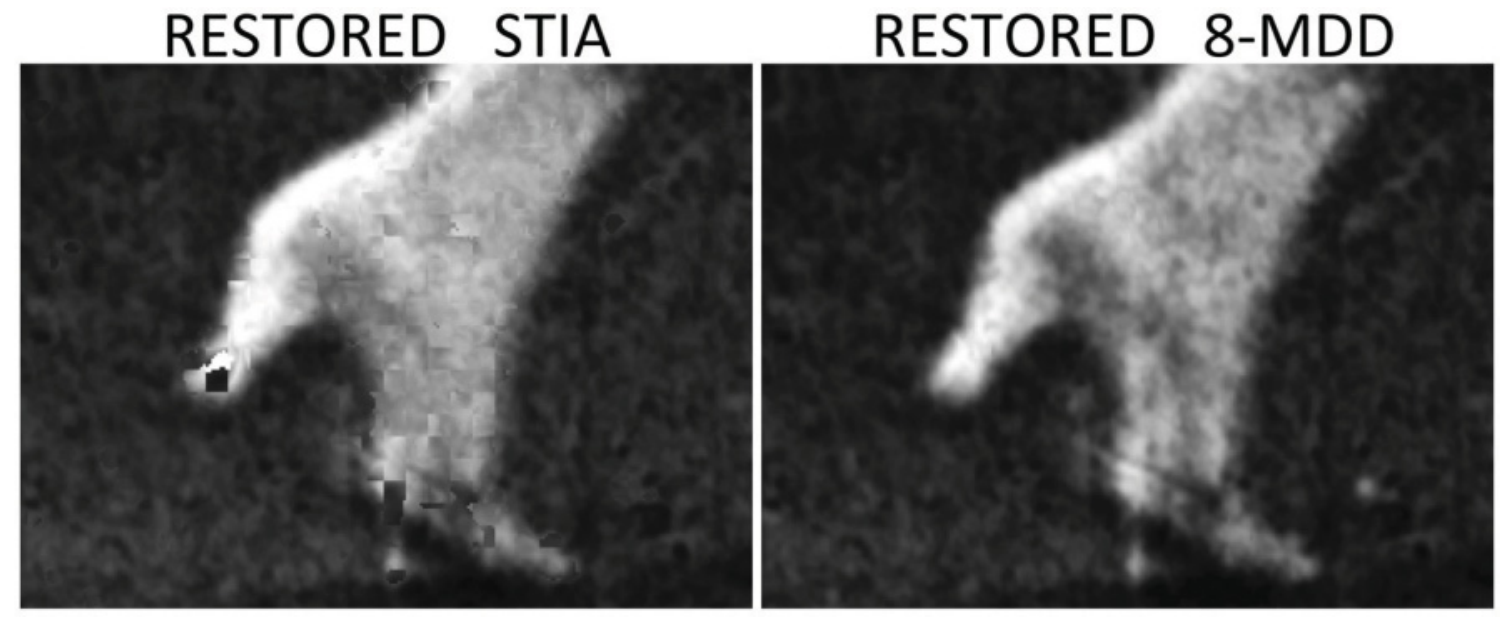

Fig. 15. Restored images: Comparison between STIA and 8-MDD. STIA produces artifacts on fast elements.

The examination of the restored sequences reveals that the motion of the scene is not the only problem afflicting the STIA. As a matter of fact, the imperfections of the scene stabilization and the variation of the random pattern of the film grain also constitute movements that produced many false detections. In the movie version restored with STIA, the film grain was partially removed; in this respect, the adoption of a higher image resolution may be unfavorable, since the grain pattern is better resolved and its variation between frames makes the images differ more.

\section{CONCLUSIONS}

In the present work, we defined in detail three "optical" methods for automatic detection of dust and scratches in the digital reproductions of photographic film. The methods presented, which are applicable on any type of transparent photographic material, are indicated as

-Polarized Dark-field Detection (PDD);

-Dual Collimation Detection (DCD); and

-Multiple Direction Detection ( $n-M D D)$.

ACM Journal on Computing and Cultural Heritage, Vol. 8, No. 2, Article 7, Publication date: March 2015. 
In light of the results of the comparison between the performances of these methods, and considering the state-of-the-art digital restoration, $n$-MDD stands out as a particularly innovative and promising technique, applicable on silver-based as well as on dye-based material, on still images as well as on moving images. The method consists in the extraction of the differences between $n$ images acquired with different illumination directions.

The 8-MDD (with eight different illumination directions) was compared with the STIA, which is the conventional approach for automatic dust and scratches detection on silver-based material. The numerical result of the comparison demonstrated that the 8-MDD outperformed the STIA.

The comparison also highlighted the main weakness of the two methods: On the one hand, the optical 8-MDD misses all the blotches due to flaws that were impressed in the film during previous copying/printing operations; on the other hand, the nonoptical STIA produces severe artifacts due to (I) the scene motion, (II) imperfections of the scene stabilization and (III) the unsteady grain pattern, which was erroneously removed during restoration.

For superficial scratches, which do not obliterate the underlying area, it was noticed that 8-MDD is sometimes too sensitive and it overdetects; however, 8-MDD completely avoids outright blunders, and this is a paramount benefit in the framework of the restoration of cultural property.

In conclusion, the MDD can be acknowledged as a very effective method; nonetheless, no general solution is attainable that is effective for all types of local flaws. A comprehensive automatic flaw detection needs to involve and cleverly combine different methods, each of them detecting a specific class of flaws.

\section{ACKNOWLEDGMENTS}

The authors wish to thank Andreas Wassmer (Digital Humanities Lab - Universität Basel) for his key support in developing the SAFRAN software, Professor Sabine Susstrunk and Dominic Rüfenacht (Images and Visual Representation Group - EPFL), partners of the project, for their important contribution in the delineation of the detection methods.

\section{REFERENCES}

J. H. Altman. 1977. Sensitometry of black-and-white materials. The Theory of the Photographic Process (4th ed.). Macmillan.

P. Baldi, S. Brunak, Y. Chauvin, C. A. Andersen, and H. Nielsen. 2000. Assessing the accuracy of prediction algorithms for classification: An overview. Bioinformatics 16, 5 (May 2000), 412-424.

A. E. Battles, D. M. Bloom, D. K. Campbell, O. R. Herrera E., and D. J. Stavely. 1999. Film scanner with dust and scratch correction by use of dark-field illumination. U.S. Patent No. 5,969,372 A. (Oct. 1999).

W. Benjamin. 1963. Das Kunstwerk im Zeitalter seiner technischen Reproduzierbarkeit: Drei Studien zur Kunstsoziologie. Suhrkamp Verlag, Frankfurt am Main, D.

R. Bergman, R. Maurer, H. Nachlieli, G. Ruckenstein, P. Chase, and D. Greig. 2008. Comprehensive solutions for removal of dust and scratches from images. J. Electron. Imaging 17, 1 (2008), 013010.

M. Bertalmìo. 2006. Strong-continuation, contrast-invariant inpainting with a third-order optimal PDE. IEEE Trans. Image Process. 15, 7 (July 2006), 1934-1938.

C. F. Bohren and D. R. Huffman. 1983. Absorption and Scattering of Light by Small Particles. John Wiley \& Sons, New York, NY.

V. Bruni and D. Vitulano. 2004. A generalized model for scratch detection. IEEE Trans. Image Process. 13, 1 (Jan. 2004 ), 44-50.

O. Buisson, S. Boukir, and B. Besserer. 2003. Motion compensated film restoration. Mach. Vision Appl. 13 (2003), $201-212$.

A. Callier. 1909. Absorption and scatter of light by photographic negatives. J. Phot. 33 (1909), 200-210.

D. Coffin. 2013. DCRAW VERSION 9.19. Retrieved from http://www.cybercom.net/dcoffin/dcraw/dcraw.c.

D. Corrigan, N. Harte, and A. Kokaram. 2008. Pathological motion detection for robust missing data treatment. EURASIP J. Adv. Signal Process. 542436 (April 2008), 1-16.

A. Crawford, V. Bruni, A. Kokaram, and D. Vitulano. 2007. Multi-scale semi-transparent blotch removal on archived photographs using Bayesian matting techniques and visibility laws. In Proceedings of the IEEE International Conference on Image Processing (ICIP'07). 
A. D. Edgar. 1993. System and method for image recovery. U.S. Patent No. 5,266,805. (November 1993).

M. A. Elgharib, F. Pitié, and A. Kokaram. 2013. Blotch and scratch removal in archived film using a semi-transparent corruption model and a ground-truth generation technique. EURASIP J. Image Video Process. 33 (June 2013), 1-20.

FIAF. 2013. Code of Ethics. Retrieved from http://www.fiafnet.org.

N. Ghosh, A. Pradhan, P. K. Gupta, S. Gupta, V. Jaiswal, and R. P. Singh. 2004. Depolarization of light in a multiply scattering medium: Effect of the refractive index of a scatterer. Phys. Rev. E 70, 6 (Dec. 2004), 066607.

R. C. Gonzalez and P. Wintz. 1987. Digital Image Processing (2nd ed.). Addison-Wesley, Reading, MA.

R. Gschwind. 2010. Dust BW. In JTS 2010-Digital Challenges and Digital Opportunities in Audiovisual Archiving. Joint Technical Symposium, Oslo, Norway.

R. Gschwind, L. Rosenthaler, A. Wittmann, W. Graff, and A. Gunzinger. 1998. Restoration of old movie films by digital image processing. In Care of Photographic, Moving Image and Sound Collections Conference, Susie Clark (Ed.). York, UK, 150-155.

M. K. Güllü, O. Urhan, and S. Ertürk. 2006. Scratch detection via temporal coherency analysis and removal using edge priority based interpolation. In Proceedings of the IEEE International Symposium on Circuits and Systems (ISCAS'06). 4591-4594.

A. Hornberg. 2006. Handbook of Machine Vision. Wiley, New York.

L. Joyeux, O. Buisson, B. Besserer, and S. Boukir. 1999. Detection and removal of line scratches in motion picture films. In Proceedings of IEEE International Conference on Computer Vision and Pattern Recognition (CVPR'99). 548-553.

D. Keeling. 1972. Optical design and aberrations. Brit. J. Phot. 119, 5837 (1972).

A. Kokaram. 1998. Motion Picture Restoration: Digital Algorithms for Artifact Suppression in Degraded Motion Picture Film and Video. Springer, New York.

A. Kokaram. 2004. On missing data treatment for degraded video and film archives: A survey and a new Bayesian approach. IEEE Trans. Image Process. 13, 3 (March 2004), 397-415.

K. Kreimeier. 1999. The Ufa Story: A History of Germany's Greatest Film Company, 1918-1945. University of California Press, Los Angeles, CA.

V. Kwatra, I. Essa, A. Bobick, and N. Kwatra. 2005. Texture optimization for example-based synthesis. ACM Trans. Graph., SIGGRAPH 2005 24, 3 (August 2005).

G. V. Landon. 2013. Automatic photometric restoration of historical photographic negatives. In Proceedings of the 2nd International Workshop on Historical Document Imaging and Processing (HIP'13). ACM, New York, NY, 1-7.

G. V. Landon, D. Clarke, and W. B. Seales. 2009. A new technique for the digitization and restoration of deteriorated photographic negatives. EURASIP J. Image Video Process. 217016 (October 2009), 1-13.

T. W. Mead. 2007. Optical scatter correction for film scanners. U.S. Patent No. 2007/0076275 A1. (April 2007).

M. I. Mishchenko and L. D. Travis. 2000. Polarization and depolarization of light. In Light Scattering from Microstructures. Springer-Verlag, Berlin. 159-175.

A. Newson, A. Almansa, Y. Gousseau, and P. Pérez. 2013. Temporal filtering of line scratch detections in degraded films. In IEEE International Conference on Image Processing (ICIP 2013). 4088-4092.

A. Newson, P. Pérez, A. Almansa, and Y. Gousseau. 2012. Adaptive line scratch detection in degraded films. In Proceedings of the 9th European Conference on Visual Media Production (CVMP'12). Association for Computing Machinery, New York, NY, 66-74.

G. Palma, M. Corsini, P. Cignoni, R. Scopigno, and M. Mudge. 2010. Dynamic shading enhancement for reflectance transformation imaging. J. Comput. Cultural Heritage 3, 2 (Sept. 2010), 6:1-6:20.

R. Pintus, T. Malzbender, O. Wang, R. Bergman, H. Nachlieli, and G. Ruckenstein. 2010. Photo repair and 3D structure from flatbed scanners using 4- and 2-source photometric stereo. In Computer Vision, Imaging and Computer Graphics. Theory and Applications. Communications in Computer and Information Science, Vol. 68. Springer, Berlin.

D. Rüfenacht, G. Trumpy, R. Gschwind, and S. Süsstrunk. 2013. Automatic detection of dust and scratches in silver halide film using polarized dark-field illumination. In Proceedings of the 2013 IEEE International Conference on Image Processing. IEEE Signal Processing Society.

J. Sassoon. 2007. Photographic meaning in the age of digital reproduction. Arch. Soc. Stud. J. Interdiscipl. Res. 1, 0 (March 2007).

P. Schallauer, A. Pinz, and W. Haas. 1999. Automatic restoration algorithms for $35 \mathrm{~mm}$ film. J. Comput. Vis. Res. - MIT 1, 3 (1999), 60-85.

P. Soille. 2003. Morphological Image Analysis. Springer, Berlin.

R. Storey. 1985. Electronic Detection and Concealment of Film Dirt. Technical Report BBC RD 1985/4. BBC.

J. G. Streiffert. 1947. Callier Q of various motion picture emulsions. J. Soc. Mot. Pict. Engrs. 49, 6 (Dec. 1947), 506-522.

A. Swan. 1981. Conservation of photographic print collections. Library Trends 30, 2 (1981), 267-296.

ACM Journal on Computing and Cultural Heritage, Vol. 8, No. 2, Article 7, Publication date: March 2015. 
The Foundry. 2011. User Guide for Furnace 4.2 for Nuke. Technical Report. The Foundry Visionmongers Ltd.

G. Trumpy. 2013. Multi-directional illumination for automatic flaw detection on photographic film: The importance of being many. In Proceedings of the Colour and Visual Computing Symposium 2013 (CVCS'13). Institute of Electrical and Electronics Engineers, Gjøvik, Norway.

G. Trumpy and R. Gschwind. 2012. Computational photography for dust and scratch detection on transparent photographic material. In SMPTE 2012 Annual Technical Conference \& Exhibition. Society of Motion Picture and Television Engineers, Hollywood, CA.

G. Trumpy, A. Wassmer, and R. Gschwind. 2011. DUST BW: Detection of dust and scratches on photographic silver halide material by dark field illumination and crossed polarization. In Proceedings of Electronic Imaging \& the Visual Arts. EVA, Berlin.

C. Tuttle. 1926. The relationship between diffuse and specular density. J. Opt. Soc. Am. 12, 6 (1926), 559-565.

T. Vitale. 2009. Film Grain, Resolution and Fundamental Film Particles. Retrieved from http://www.vitaleartconservation.com.

R. Wallis. 1976. An approach to the space variant restoration and enhancement of images. In Proceedings of Symposium on Current Mathematical Problems in Image Science. Naval Postgraduate School, Monterey, CA, 329-340.

R. Wright and A. Williams. 2001. Archive Preservation and Exploitation Requirements. Survey. PRESTO-Preservation Technologies for European Broadcast Archives.

B. Xiao. 2010. Principal Component Analysis for Feature Extraction of Image Sequence, Vol. 1. Computer and Communication Technologies in Agriculture Engineering (CCTAE).

Received November 2013; accepted July 2014 